\title{
Valoración de resultados y perfil psicosocial de un programa de rehabilitación de personas con drogodependencias
}

\author{
Luis Valero-Aguayo*, Margarita Ortiz-Tallo, María del Mar Parra-García y Marta Jiménez-Guerra \\ Departamento de Personalidad, Evaluación y Tratamiento Psicológico, Facultad de Psicología, Universidad de Málaga, España
}

\begin{abstract}
Resumen: Se describen los resultados y el perfil psicosocial de las personas atendidas durante los últimos 3 años en un programa de rehabilitación en drogodependencias del Proyecto Hombre en Málaga. La muestra seleccionada ha sido de 96 personas entre 18 y 56 años $(M=31.31, D T=14.20)$, el $83.3 \%$ hombres y $16.7 \%$ mujeres. El porcentaje de altas terapéuticas se sitúa en el $22.9 \%$. Se han estudiado las diversas características psicosociales, relacionándolas con la probabilidad de alta, abandono o recaída en adicciones. Se detallan los resultados y análisis estadísticos en torno a características personales, familiares y de consumo. Se ha encontrado como factor fundamental relacionado con los abandonos: el policonsumo, el abuso emocional y físico, y especialmente la cantidad de conflictos familiares. Se sugieren orientaciones terapéuticas que mejoren los resultados y resuelvan los conflictos familiares que podrían ser un factor importante en las recaídas y abandonos del programa.

Palabras clave: Drogodependencia; valoración de programas; proyecto hombre; perfil psicosocial.
\end{abstract}

Title: Evaluation results and psychosocial profile of a rehabilitation program for people with drug abuse.

Abstract: This research group carried out an evaluation of data collected over the last 3 years, from the drug addiction rehabilitation program Proyecto Hombre Málaga. The sample consisted of 96 participants from 18 to 56 years old $(M=31.31, D T=14.20)$, i.e., $83.3 \%$ male and $16.7 \%$ female. The rate of success, those who finished the therapeutic program successfully, was $22.9 \%$. The various psychosocial characteristics were studied, and related to the likelihood of therapeutic success, dropouts and relapse back into addiction. Details of the results and statistical analysis of the personal characteristics, family and consumer products are shown. The principal factors related to dropouts were found to be: poly-drug use, physical and emotional abuse and especially, frequent family conflicts. Therapeutic guidelines are suggested which improve the overall results and help resolve family conflicts that could be an important factor for relapse and for dropping out of the program.

Keywords: Drug abuse; program evaluation; proyecto hombre; psicosocial features.

\section{Introducción}

Identificar un perfil psicosocial en el que se destaquen posibles factores de riesgo, de vulnerabilidad o de protección relacionados con el éxito de la intervención en drogodependencias es de gran importancia para la mejora de los programas. En primer lugar de los propios programas de tratamiento, para poder diseñar estrategias distintas de rehabilitación y reinserción encaminadas a las necesidades específicas de los usuarios y sus familias. Por otro lado, en el campo de la prevención, para dirigir la aplicación práctica en programas educativos en aquellos jóvenes y en las familias que presenten un mayor riesgo de consumo y de recaída. La Sociedad Española de Toxicomanía reconocía la importancia de la investigación en drogodependencias, además de resaltar la colaboración entre instituciones y los distintos tipos de investigación más básica, epidemiológica, clínica, preventiva o social. En uno de sus puntos resaltaba la importancia de la difusión de los resultados de las investigaciones, la transferencia del conocimiento más clínico, del día a día, hacia el resto de investigadores y personal que trabaja en este campo; y también sobre la eficacia de las estrategias terapéuticas y tratar de identificar los factores de riesgo y protección sobre la dependencia a las drogas (Rodríguez et al., 2006; SET, 2006).

Sin embargo, la investigación sobre resultados terapéuticos no es tan fácil ni abundante en la literatura sobre el tema, menos aún si se trata de estudios a largo plazo. Girón (2007) señala estas dificultades al analizar las publicaciones de valo-

* Dirección para correspondencia [Correspondence address]: Luis Valero Aguayo, Departamento Personalidad, Evaluación y Tratamiento Psicológico, Facultad de Psicología, Campus Teatinos, Universidad de Málaga, 29071 Málaga (España).Email: lvalero@uma.es ración de programas de intervención en drogodependencias, por la poca rigurosidad metodológica, la eliminación de los datos de usuarios no entrevistados, la no consideración de recaídas, y el uso exclusivo de datos basados en entrevista y autoinformes. Este tipo de estudios se basa en identificar y entrevistar a una muestra lo más amplia posible de personas que han pasado por un tratamiento de desintoxicación determinado, y evaluar fundamentalmente su situación de noconsumo y otras variables familiares, laborales, económicas, judiciales, salud, etc., que pudiesen predecir de alguna forma los resultados exitosos o no tras pasar por el programa de tratamiento. Otros estudios más experimentales comparan la eficacia entre distintos tipos de programa de desintoxicación, o frente a grupos de control o cohortes de muestras similares que no hayan pasado por esos programas. Según esta revisión (Girón, 2007) habría sólo 5 estudios más rigurosos sobre seguimiento, que ofrecen unos resultados generales: reducción en el uso de drogas entre el 25 y $38 \%$, sobre todo la cocaína o la droga principal del usuario, y en porcentajes menores en el alcohol, cannabis y el uso de varias drogas simultáneas; habría porcentajes mayores en los programas residenciales de larga duración (entre 50-60\%) o en los de mantenimiento de metadona (sobre 69\%); informan de la disminución de otros problemas tales como menos actividades delictivas, mayores niveles de empleo y mayor nivel económico que el que tenían los usuarios al comenzar el programa; habría mejorías en el estado de salud física y mental de los usuarios en ese seguimiento, reducción de hospitalizaciones y menos conductas de riesgo. Otros estudios a largo plazo, entre 1 y 3 años, han encontrado resultados similares sobre la reducción de delitos, mejoras en la salud y prevención de SIDA (Vanderplasschen, Bloor y McKeganey, 2010; White, Browne y McKiernan, 2010), aunque esos éxi- 
tos se ven reducidos en aquellos programas estatales u obligatorios por orden judicial (Evans, Li y Hser, 2008).

Entre los posibles factores predictores de los resultados en esos seguimientos, esta revisión de estudios (Girón, 2007) encontró que la mayor permanencia en tratamiento, por mas de un año, aseguraba unos resultados más favorables a largo plazo (especialmente con los programas de mantenimiento de metadona), y también en los programas de comunidad terapéutica, mientras que los usuarios en programas ambulatorios disminuían sus probabilidades de buenos resultados futuros. Entre esas variables importantes, también encontraron que el uso intensivo de alcohol hacía más probable volver a consumir tras el tratamiento. En general, cuanto mayor consumo antes, mayor es la probabilidad de seguir consumiendo después. Las mujeres suelen tener mejores índices de inserción, con menos delincuencia y más probabilidad de empleo tras el tratamiento. Encontraron que la depresión y el trastorno antisocial eran los problemas mentales más relacionados con la recaída y continuar el uso de drogas tras el tratamiento. Mientras que la obtención de un empleo o estabilidad económica son predictores del mantenimiento a largo plazo libre de drogas. Las personas con más experiencias terapéuticas anteriores y con más problemas de consumo, eran las que tenían peores resultados. Y los que no terminaban los tratamientos aumentaban sus posibilidades de recaer significativamente. Como factor personal, cuando la motivación es extrínseca a partir de los familiares o por razones judiciales, las esperanzas de éxito son menores que si es por propia decisión y motivación intrínseca. En este sentido, la percepción positiva sobre los terapeutas es una variable significativa sobre el mayor tiempo de permanencia en el tratamiento y mejor funcionamiento mental posterior. Un estudio comparativo entre nueve programas de desintoxicación norteamericanos ha mostrado que una variable predictiva importante es el número de sesiones terapéuticas en el primer mes para motivar y comprometer al usuario en esa abstinencia (Crevecoeur et al., 2010). Otros autores han encontrado variables predictivas en la satisfacción con los terapeutas y el centro (Arbour, Hambley y Ho, 2011), el policonsumo unido a cocaína (Hambley, Arbour y Sivagnanasudaram, 2010), la presencia de trastornos mentales y depresión, junto con mayor dependencia de hipnóticos y estimulantes (Mulder, Frampton, Peka, Hampton y Masters, 2009),

Los programas publicados en España muestran datos similares, aunque sean con muestras más pequeñas y sin comparaciones con grupos control. Aparecen índices entre 30 y 56\% de situación favorable después de 6 y 12 meses tras terminar el tratamiento. Se encuentran correlaciones positivas entre el tiempo dentro del programa y los mejores resultados, menores actividades delictivas y mejor integración social; y se repite el hecho de que a mayor gravedad de la adicción y mayor historia, peor pronóstico en los resultados. Entre los estudios de programas españoles, el Proyecto Hombre fue evaluado en 2001 por Fernández y Secades (2001) en las comunidades autónomas de Asturias, Baleares y Galicia, con una muestra de 452 entrevistas. De ellos, el
$80 \%$ habían terminado el programa completo con alta terapéutica, y un $84 \%$ continuaban en abstinencia de drogas, incluyendo algunos que habían abandonado el programa antes de terminar; y habían disminuido considerablemente los incidentes legales o actividades delictivas tras el tratamiento. En el estudio de Secades y Benavente (2000) específicamente sobre los posibles factores predictores de la retención dentro de la comunidad terapéutica, encontraron que la tasa media de abandonos era del $32.7 \%$ con una permanencia media de 7 meses en el programa, y que esa tasa iba disminuyendo conforme pasaban los meses dentro del programa. Entre las variables más relevantes encontraron las relacionadas con el propio programa, sus exigencias, consumos durante el tratamiento y los familiares que prestan apoyo durante ese tiempo.

López-Goñi et al. (2008a, 2008b) han publicado un estudio sobre el Proyecto Hombre en Navarra analizando las variables que inciden en los abandonos dentro del programa. Entrevistaron a 61 usuarios sobre las razones de su abandono y encontraron que el $70.5 \%$ eran debidos a las limitaciones impuestas por el propio programa terapéutico, en el $39.3 \%$ por el cansancio dentro de los grupos, el $34.4 \%$ por deseos de consumir droga de nuevo, y el $49.2 \%$ por haber conseguido sus objetivos personales, pero también abandonaban por malas relaciones con otros usuarios (13.1\%) y mala relación con los terapeutas (19.7\%). Otro estudio de este mismo equipo (Fernández-Montalvo et al., 2008) con mayor cantidad de entrevistados, encontró diferencias entre los usuarios que habían abandonado el programa en cuanto a las variables como reducción de conductas criminales y mejoras en la salud. Aunque en estos estudios no se han relacionado los abandonos con otras variables personales o familiares, es mayoritaria la opinión de que las condiciones estrictas que han de cumplir los usuarios dentro del programa es una de las razones fundamentales para los abandonos, que ya aparecía en otro estudio de valoración anterior en la comunidad de Asturias (Fernández-Hermida et al., 1999).

El estudio de Sánchez-Hervas et al. (2010) en varios centros ambulatorios de Valencia sobre los posibles factores predictivos de los abandonos en personas adictas a la cocaína, sí encontró diferencias significativas en lo que denominan "gravedad adictiva", es decir, cuanta más historia en la adicción y mayor cantidad de sustancias, también mayor abandono prematuro del tratamiento en los tres primeros meses.

En relación a este conjunto de variables que parecen influir en el mantenimiento de los resultados de los tratamientos en drogodependencias, y más específicamente las variables relacionadas con la retención en los programas de tratamiento residenciales en comunidades terapéuticas, el presente estudio pretende: (1) por un lado, presentar un perfil psicosocial de los usuarios adultos que han recibido tratamiento en un centro del Proyecto Hombre en Málaga, y (2) analizar los posibles factores de protección o de vulnerabilidad en función de las características del consumo, la situación personal y los factores familiares que puedan relacio- 
narse con el éxito del tratamiento, con alta terapéutica, o el abandono temprano del mismo.

\section{Método}

Se ha llevado a cabo una evaluación externa desde el Grupo de Investigación HUM-300 sobre "Valoración de programas sociales", a petición del Proyecto Hombre Málaga. Este grupo había realizado ya una evaluación cualitativa en los años iniciales del centro (Cardenal, Moreno y Ortiz-Tallo, 1994). El programa terapéutico de Proyecto Hombre tiene unas líneas generales con una base filosófica e inspiración de psicoterapia humanista, intentando la rehabilitación de los usuarios de forma global, como personas sociales. De hecho mezcla muy distintas técnicas psicológicas. Como programa general diferencia tres fases principales: la primera fase es la denominada "Acogida", de tipo ambulatorio, que dura entre varios meses y un año, que tiene por objetivos superar el síndrome físico de abstinencia, desarrollar la motivación para cambiar y mejorar el concepto de sí mismo como persona. La segunda fase es la denominada "Comunidad", también entre nueve meses y un año, realizada en un centro comunitario en las afueras de la ciudad, donde el usuario convive diariamente con otros usuarios y terapeutas, y cuyo objetivo es el adquisición de hábitos diarios y relaciones personales, con objeto de superar sus problemas y crear valores humanos que le lleven a mantenerse libre de drogas fuera de la comunidad posteriormente. $\mathrm{Al}$ final de ésta los responsables dan el "alta terapéutica". Una tercera fase es la "Inserción sociolaboral" desarrollada junto con las anteriores y cuyo objetivo es adquirir un estilo de vida autónomo, buscando metas propias y encontrar un trabajo con el que independizarse y seguir su vida autónoma fuera del programa. Para más detalles e información sobre las características de Proyecto Hombre puede consultarse su web www.proyectohombre.es.

El objetivo en este estudio es describir el perfil psicosocial de la población a la que atienden, los resultados de la intervención en relación a tasas de abandono y finalización del programa, y las posibles variables relacionadas de esos resultados. Se realizaron varias reuniones con objeto de conocer las formas de recogida de datos, tipos de informaciones y proceso para llevar a cabo esa evaluación. El número de participantes es el total de casos recopilados por vía informática. Se toma esta decisión tras comprobar la dificultad que supone acceder a muchos de los cuestionarios originales en formato papel de los últimos tres años. De esta forma, la muestra tomada de 96 participantes representa aproximadamente el $25 \%$ del total de la población atendida en el centro de adultos.

Se trata de una investigación post-facto, pues los datos se han tomado a partir de los datos ya acumulados en los historiales del centro. Se han utilizado las entrevistas iniciales y los historiales personales, realizados por las dos psicólogas del centro durante las dos primeras semanas cuando entraban los usuarios al centro por primera vez. Algunos de los ítems se han agrupado en categorías si/no (presencia/ausencia), y en otros casos como categorías ordinales, con objeto de realizar análisis estadísticos en todos los indicadores posibles. También se ha utilizado el cuestionario Registro Inicial Familiar, una entrevista semiestructurada para adolescentes y familiares con formato de cuestionario realizada por el propio centro (Secades y García, 2005) que agrupa los contenidos respecto a lo informado por los padres y otros adultos, problemática percibida, área familiar, área académico-laboral, área social, valoración de los padres y valoración del entrevistado. Además se han utilizado los datos del EuropASI, un cuestionario conjunto como entrevista semi-estructurada y autoinforme que evalúa distintas áreas de los problemas adictivos tales como salud física, empleo y recursos, drogas y alcohol, situación legal, historia familiar, relaciones familiares y sociales, y salud mental. Todas las subescalas tienen una parte autoaplicada por el propio usuario, la valoración subjetiva del entrevistador y el grado de validez de la información obtenida. Todo ello como un índice de gravedad de la adicción. Existen versiones para distintos países europeos, donde se han obtenido índices de fiabilidad test-retest entre .47 y .93 , y fiabilidad de alpha de Cronbach entre .69 y .92 (Bobes et al., 2007).

Los datos se iban recogiendo en un fichero informatizado, con categorías y códigos, manteniendo el anonimato de los datos para impedir una identificación personal. Se ha utilizado el programa SPSS-16 para obtener descriptores estadísticos; análisis de categorías con $C h i^{2}$ combinando las distintas variables, especialmente al contrastar con los resultados del programa (altas y abandonos); análisis de varianza con aquellas variables continuas que lo permitían; y análisis correlacionales respecto a variables como edad o comienzo del consumo de las distintas sustancias.

\section{Resultados}

\section{Características de los participantes}

La muestra para el estudio ha sido seleccionada del total de las personas atendidas en el Proyecto Hombre Málaga en los últimos tres años, debiendo cumplir los requisitos de tener más de 18 años de edad y haber acudido al centro por tener problemas con el consumo habitual de drogas.

En este estudio han participado 96 usuarios adultos (83.3\% hombres y $16.7 \%$ mujeres) que han acudido entre los años 2007 a 2009 a la Asociación Proyecto Hombre de Málaga. Las edades de los participantes están comprendidas entre los 18 hasta los 56 años ( $M$ edad 31.31, DT=12.4) y todos presentan problemas de abuso de drogas. En relación a la curva de población normal, hay más usuarios de 25-30 años y de 40 años que la distribución habitual de la población andaluza.

En relación al estado civil el 76\% de ellos están solteros, el 7.3\% casados, el $11.5 \%$ separados y el 5.2\% divorciados. $\mathrm{Si}$ atendemos a las personas con las que en el momento del estudio están conviviendo, encontramos que el $35.4 \%$ vive 
con sus padres, el 15. $6 \%$ con otros familiares, el $13.5 \%$ con su pareja, otro $13.5 \%$ con su pareja e hijos, el $11.5 \%$ vivían en situación inestable, el 5.2\% solos, el $4.2 \%$ con amigos y, por último un $1 \%$ en un medio protegido.

Tabla 1. Características sociológicas de la muestra.

\begin{tabular}{|c|c|c|}
\hline & Frecuencia & Porcentaje \\
\hline Hombres & 80 & 83.3 \\
\hline Mujeres & 16 & 16.7 \\
\hline \multicolumn{3}{|l|}{ Estado Civil } \\
\hline Soltero/a & 73 & 76.0 \\
\hline Casado & 7 & 7.3 \\
\hline Separado & 11 & 11.5 \\
\hline Divorciado & 5 & 5.2 \\
\hline \multicolumn{3}{|l|}{ Edad } \\
\hline Hasta 20 años & 8 & 8.3 \\
\hline 21-25 años & 28 & 29.2 \\
\hline 26-30 años & 15 & 15.6 \\
\hline 31-35 años & 8 & 8.3 \\
\hline 36-40 años & 19 & 19.8 \\
\hline 41-45 años & 15 & 15.6 \\
\hline 46-50 años & 2 & 2.1 \\
\hline 51-55 años & 0 & 0.0 \\
\hline 56-60 años & 1 & 1.1 \\
\hline \multicolumn{3}{|l|}{ Convivencia } \\
\hline Padres & 34 & 35.4 \\
\hline Pareja & 13 & 13.5 \\
\hline Pareja e hijos & 13 & 13.5 \\
\hline Familiares & 15 & 15.6 \\
\hline Amigos & 4 & 4.2 \\
\hline Medio protegido & 1 & 1.0 \\
\hline Solo & 5 & 5.2 \\
\hline No estable & 11 & 11.5 \\
\hline Personas alcohol & 9 & 9.4 \\
\hline Personas drogas & 9 & 9.4 \\
\hline Familiares con problemas & 7 & 7.3 \\
\hline Familiares sin problemas & 69 & 71.9 \\
\hline Amigos con problemas & 5 & 5.2 \\
\hline Amigos sin problemas & 12 & 12.5 \\
\hline
\end{tabular}

\section{Consumo}

En cuanto al tipo de consumo hay diversos indicadores: sobre el consumo en general, en el último mes, la edad de inicio en esas sustancias, y el número de ellas que consumen simultáneamente. Hay unos porcentajes muy elevados en el consumo de cuatro sustancias en todos los usuarios: cocaína (90.6\%), alcohol en pequeñas cantidades $(87.5 \%)$ o grandes cantidades $(76.0 \%)$ y cannabis $(81.3 \%)$. Las demás sustancias presentan porcentajes menores entre el 34 y 10\% según cada caso concreto. De hecho, al preguntarles sobre qué han consumido en el último mes vuelven a repetirse el mismo tipo de sustancias, aunque afirman porcentajes algo más bajos (cocaína $=70.8 \%$, alcohol $=56.3$ y $46.9 \%$, y cannabis $=47.9 \%$ ). Hay un $28.1 \%$ de usuarios que afirman tomar múltiples sustancias a la vez. Precisamente al reunir los datos sobre qué sustancia declara consumir cada usuario, hay unos porcentajes muy elevados que consumen 4,5 y 6 sustancias al mismo tiempo $(27.1 \%, 20.8 \%$ y $14.6 \%$ respectivamente). También ocurre algo similar en cuanto al número de sustancias consumidas en el último mes de forma simultánea (entre 2 y 4 , con $18.8 \%, 19.8 \%$ y $22.9 \%$ respectivamente). Incluso hay algunos usuarios que declaran que han consumido hasta 8 y 9 sustancias.

La edad de inicio en el consumo de las distintas sustancias es muy variada pues puede ir desde los 8 años hasta los 50 años en que afirman haberlas probado por primera vez. Sin embargo, al ordenar los datos aparece una tendencia clara en la historia del consumo, tanto si se considera la media de inicio como la mediana (como más representativo de frecuencia de usuarios). En este caso, el orden en el inicio de drogas comienza con cantidades pequeñas de alcohol, después cannabis, siguen inhalantes y alucinógenos (entre $15 \mathrm{y}$ 16 años), anfetaminas y alcohol en grandes cantidades (17-18 años), y cocaína, opiáceos, y heroína (18-20 años). Incluso la metadona aparece ya a edades más tardías, se supone que como efecto de los programas de desintoxicación anteriores a edades más adultas. Esta progresión en edad se repite de forma sistemática, por lo que podría describir una curva de adquisición de distintas sustancias, probablemente según su frecuencia de uso o su gravedad percibida.

Tabla 2. Datos sobre el consumo informado por los usuarios.

\begin{tabular}{|c|c|c|c|c|c|c|c|c|c|}
\hline & \multicolumn{2}{|c|}{ Consumo } & \multicolumn{2}{|c|}{ Último mes } & \multicolumn{2}{|c|}{ Años consumiendo } & \multicolumn{3}{|c|}{ Edad inicio } \\
\hline & $\mathrm{N}$ & $\%$ & $\mathrm{~N}$ & $\%$ & Rango & Media & Rango & Media & Mediana \\
\hline Alcohol pequeñas cantidades & 84 & 87.5 & 54 & 56.3 & $2-32$ & 16.0 & $8-50$ & 15.7 & 15 \\
\hline Cannabis & 78 & 81.3 & 46 & 47.9 & $1-32$ & 13.3 & $10-47$ & 16.2 & 15 \\
\hline Inhalantes & 11 & 11.5 & 1 & 1.0 & $1-25$ & 8.3 & $14-30$ & 18.2 & 15 \\
\hline Alucinógenos & 33 & 34.4 & 6 & 6.3 & $2-28$ & 10.6 & $13-29$ & 17.4 & 17 \\
\hline Anfetaminas & 22 & 22.9 & 4 & 4.2 & $1-31$ & 12.0 & $13-38$ & 19.8 & 18 \\
\hline Alcohol grandes cantidades & 73 & 76.0 & 45 & 46.9 & $1-32$ & 11.6 & $11-42$ & 20.3 & 18 \\
\hline Cocaína & 87 & 90.6 & 68 & 70.8 & $1-34$ & 11.0 & $8-38$ & 19.5 & 18 \\
\hline Otras sintéticas & 19 & 19.8 & 6 & 6.3 & $1-39$ & 7.2 & $13-30$ & 19.5 & 19 \\
\hline Opiáceos & 15 & 15.6 & 10 & 10.4 & $1-28$ & 9.9 & $17-43$ & 24.4 & 21 \\
\hline Benzodiacepinas & 25 & 26.0 & 15 & 15.6 & & & & & \\
\hline Heroína & 22 & 22.9 & 13 & 13.5 & $1-25$ & 12.0 & $14-31$ & 22.2 & 23 \\
\hline Metadona & 10 & 10.4 & 4 & 4.2 & $1-16$ & 4.9 & $12-38$ & 33.4 & 33 \\
\hline
\end{tabular}


Existen diversas correlaciones positivas y significativas entre la edad en que los usuarios entran en el programa, y la historia de inicio de las distintas sustancias. Así, hay una correlación entre la edad de acceso al programa y la que iniciaron la ingesta de alcohol en pequeñas o grandes cantidades $(r=.40, r=.53, p<.0001)$, el consumo de benzodiacepinas $(r=.84, p<.0001)$, cocaína $(r=.56, p<.0001)$, cannabis $(r=.36$, $p<.0001)$. Es decir, aquéllos que acceden con más edad al programa de rehabilitación también han comenzado más tarde a consumir.

También están correlacionadas las edades en que se comienza el consumo de alcohol en pequeñas o grandes cantidades con la edad en que se comienza el consumo de cannabis, cocaína, opiáceos y anfetaminas (correlaciones entre .44 y .85, $p<.05$ ). Es decir, a menor edad de comienzo con unas sustancias también menor edad en las otras, y viceversa. También se correlacionan el uso de cannabis con los alucinógenos, inhalantes y anfetaminas (correlaciones entre .49 y .84, $p<.05)$. Y, por otro lado, también se correlacionan las edades de comienzo de la heroína, los opiáceos y la cocaína; y las benzodiacepinas con la cocaína (correlaciones entre .49 y $.84, p<.05)$. De alguna forma, si se hiciese un esquema relacional entre la edad de comienzo en el uso de las distintas sustancias, el alcohol es mayoritario y significativo con casi todas las demás drogas: (1) el alcohol se relaciona a su vez con cannabis, opiáceos, anfetaminas, cocaína; (2) en un segundo grado, el cannabis con alucinógenos, anfetaminas, cocaína e inhalantes; y (3) en un tercer grado éstos con los distintos opiáceos, con la cocaína, benzodiacepinas. El uso de la metadona hay pocos usuarios y parece independiente en edad de comienzo.

\section{Edad de inicio}

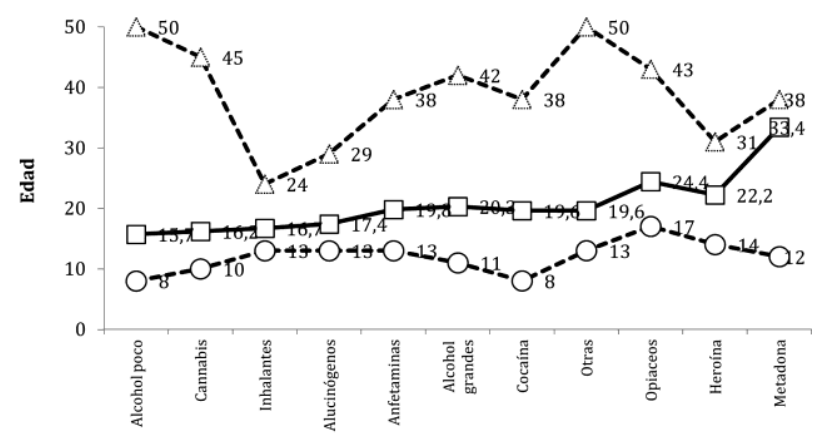

Figura 1. Edad de inicio del consumo de las distintas sustancias, incluyendo el rango menor y mayor de ese consumo.

Es también interesante la correlación negativa entre el consumo total de drogas y la edad de comienzo del abuso de alcohol y heroína $(r=-.34,-.31,-.57, p<.01)$. Es decir, a menor edad de comienzo mayor cantidad de consumo total. Y también resulta una correlación negativa entre la cantidad total de conflictos (padres, hermanos, familiares, etc.) y la edad en que comienza el abuso de grandes cantidades de alcohol y de cocaína $(r=-.32,-.29, p<.01)$. Es decir, cuando hay más conflictos personales en relación a otros, menor es la edad de inicio del consumo. Aunque sólo es una correlación, también podría concluirse que al comenzar más temprano el consumo aparecen mayor cantidad de conflictos con el entorno social inmediato.

\section{Resultados del programa}

En la muestra de este estudio hay un 57.3\% de participantes en la primera fase del programa, denominado de "Acogida" de un año aproximadamente de duración; y un $42.7 \%$ en la segunda, denominado "Comunidad" también de un año de duración. Al final de esos dos años, si han desaparecido los problemas de adicciones, dan el "alta terapéutica". No hay diferencias significativas en la proporción de personas en la primera o en la segunda fase del tratamiento, se distribuyen por igual. En cambio, sí hay diferencias en los resultados globales, no se distribuye por igual la proporción de altas terapéuticas (22.9\%), los abandonos o expulsiones del programa $(68.8 \%)$ y las recaídas o vueltas al programa una vez dado el alta (8.3\%) (Chi2 $=57.25, g l=2, p<.001)$. Aunque hay una mayor proporción de mujeres dadas de alta $(37.5 \%)$ no hay diferencias significativas en los resultados entre hombres y mujeres.

Sí hay diferencia significativas en cuando al número de abandonos y altas terapéuticas en relación a qué fase ocurren (Chi2=17.992, $g l=2, p<.001)$. Como era de esperar, en la primera fase ocurren mayor cantidad de abandonos y expulsiones $(83.6 \%)$ frente a las altas terapéuticas $(7.3 \%)$, mientras que en la segunda fase los abandonos son algo mayores $(48.8 \%)$ que las altas $(43.9 \%)$. Las recaídas se producen tanto en la primera como en la segunda fase terapéutica.

Los datos obtenidos en esta muestra concreta son paralelos a los obtenidos, en general, sobre las altas terapéuticas dentro del programa de Proyecto Hombre a lo largo de su historia. De hecho, y según sus estadísticas globales el número de altas ha ido creciendo progresivamente, pero se sitúa en los últimos años entre el 26 y el 35\%. Aunque estos porcentajes globales no consideran las recaídas en esos años, que volverían ser ingresos en el programa unos años después.

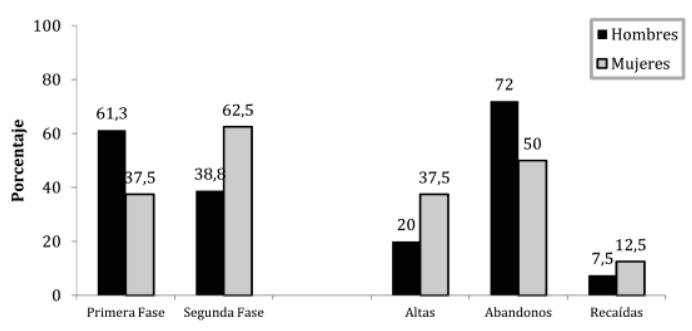

Figura 2. Resultados globales del programa en los tres últimos años. 


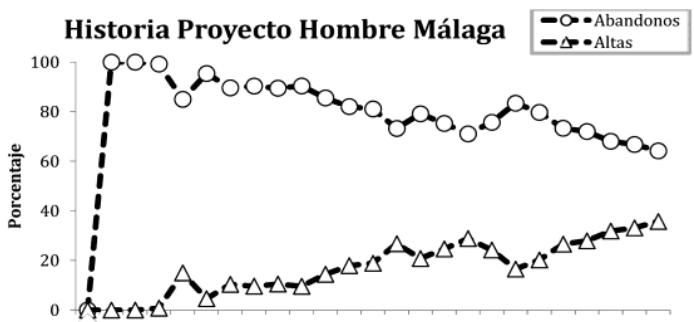

Figura 3. Evolución de abandonos y altas terapéuticas en los 25 años de historia del Proyecto Hombre Málaga.

Hay diferencias significativas respecto a los resultados, en aquéllos que consumen alcohol en pequeñas o grandes dosis, aparece siempre mayor proporción de abandonos o expulsiones (Chi2 $=10.113, g l=2, p<.01 ;$ Chi2 $=10.004, g l=2$, $p>.001)$. También aparecen mayor número de expulsiones en los que toman cannabis $(C h i 2=6.882, g l=2, p<.05)$. En el caso del consumo de heroína $(C h i 2=6.797, g l=2, p>.05)$, y el de anfetaminas (Chi2 $=9.866, g l=2, p<.01)$ e inhalantes (Chi2=7.327, $g l=2, p<.01)$ también hay diferencias, pero los datos se agrupan en los que NO-consumen y abandonan. Tampoco hay diferencias significativas en el consumo de otras sustancias como metadona, opiáceos, heroína, benzodiacepinas, barbitúricos, cocaína, alucinógenos, tanto en consumo general como en el último mes antes de entrar al programa. En suma, se podría afirmar que el consumo de alcohol en pequeñas o grandes dosis, y los que toman cannabis tienen mayor probabilidad de abandonar el programa. En cambio las demás drogas no presentan diferencias significativas respecto a los abandonos.

\section{Características psicosociales}

En general viven con los padres y otros familiares (total $51.0 \%$ ), y en menor medida tienen su propia pareja e hijos $(27.0 \%)$. También en su mayoría afirman que viven en familias sin problemas $(71.9 \%)$ y mantienen de forma asidua el contacto familiar $(46.9 \%)$. Son bastante menos los que declaran vivir con familias o amigos con problemas. El hecho de vivir con alguien que consuma alcohol o drogas, el tipo de convivencia, estado civil, tiempo de esa convivencia, y con quién pase el tiempo libre, son variables que no diferencian los resultados terapéuticos.

Sin embargo, declaran una gran cantidad de conflictos con los distintos miembros de la familia: padre $(69.8 \%)$, madre $(66.7 \%)$, pareja $(75.1 \%)$, hermanos $(53.7 \%)$, incluso otros familiares $(37.5 \%)$ y otros amigos y compañeros $(38.5 \%)$. El grado de conflicto parece elevado en todos ellos. $\mathrm{Al}$ preguntarles sobre la existencia de esos conflictos en el último mes, las cifras disminuyen aunque se mantienen las proporciones: padre $(33.3 \%)$, madre $(28.1)$, pareja $(22.9 \%$, hermanos $(30.5 \%)$, otros familiares $(13.5 \%)$, y amigos, compañeros y vecinos también tienen conflictos con ellos.

Al relacionar esta variable con los abandonos del programa, el hecho de tener conflicto con la madre resulta signi- ficativo para los abandonos (Chi2 $=7.909, g l=2, p<.05)$, también los conflictos con hermanos (Chi2 $=6.848, g l=2, p<.05)$, y con otros familiares (Chi2 $=13.592, g l=2, p<.001)$; pero no ocurre con el padre, la pareja, los hijos, los amigos, compañeros o los vecinos.

En relación a la variable antecedentes familiares el 57.3\% de los padres han tenido problemas con el alcohol, el 58.3\% con las drogas y el $42.7 \%$ problemas psiquiátricos. Sólo el $28.1 \%$ de la muestra no tiene antecedentes familiares de consumo. El 13.5\% refiere tener antecedente de uno de los problemas, el $30.2 \%$ dos de ellos y un $28.1 \%$ tiene antecedentes familiares de los tres tipos.

$\mathrm{Si}$ atendemos a los resultados terapéuticos de alta o abandono final, los antecedentes familiares, bien sea por consumo de alcohol, drogas o problemas psiquiátricos no presentan diferencias significativas. Tampoco el hecho de vivir con alguien que tome alcohol o drogas, el tipo de convivencia, estado civil, tiempo de esa convivencia, y con quién pase el tiempo libre, son variables que se diferencien respecto a los resultados terapéuticos.

Resulta curioso que aquéllos que apenas mantienen contacto familiar, tienen comparativamente más altas terapéuticas $(41.2 \%)$, y por el contrario los que sí tienen esa relación familiar estrecha tienen más abandonos y recaídas $(82.2 \%)$ (Chi2 $=23.368, g l=2, p<.0001)$. Podría ocurrir que ese contacto familiar, y los conflictos especialmente con la madre, hermanos y otros familiares (que son los significativos) sean bien un factor previo a las adicciones, o quizás un efecto posterior de esa misma problemática, e incida negativamente en los abandonos.

Al comparar los distintos grupos según sus resultados (altas, abandonos, recaídas) sólo hay diferencias significativas respecto a la variable de "conflictos totales". En este caso, en el grupo de abandonos hay mayor número de conflictos globales que aquellos a quienes se les ha dado el alta terapéutica $(M=4.76$ y $M=2.90$ respectivamente $) \quad(F=6.567$, $S C=56.07, g l=93.2, p=.002)$. No hay diferencias respecto a los abusos totales (emocionales, físicos o sexuales), ni respecto al consumo total, ni respecto a los antecedentes familiares. Parece que la acumulación de conflictos de todo tipo (familiares, pareja, vecinos, amigos, etc.) puede ser un factor influyente para un mayor abandono, aunque también podría ser un efecto posterior a la propia adicción.

Los usuarios informan sobre elevados índices de abuso emocional (68.8\%), físico (34.4\%) y sexual (11.5\%). Sólo un $28.1 \%$ informa de ningún abuso, y algunos informan de dos y tres tipos de abusos (26.0 y 8.3\%). Si atendemos a esta variable en función del sexo se encuentran diferencias significativas entre hombres y mujeres en el abuso físico sufrido. Aunque los datos son desiguales, hay una diferencia mayor en mujeres que han tenido episodios de abusos físicos $(27.5 \%$ en hombres frente a $68.8 \%$ en mujeres, Chi $2=10.057$, $p<.01) ; y$ también en abusos sexuales donde hay menos hombres $(3.8 \%)$ que mujeres $(50 \%)$ con esta historia de abuso sexual $(C h i 2=28.112, p<.001)$. No hay diferencias signifi- 
cativas en esta variable respecto al éxito o abandono del programa.
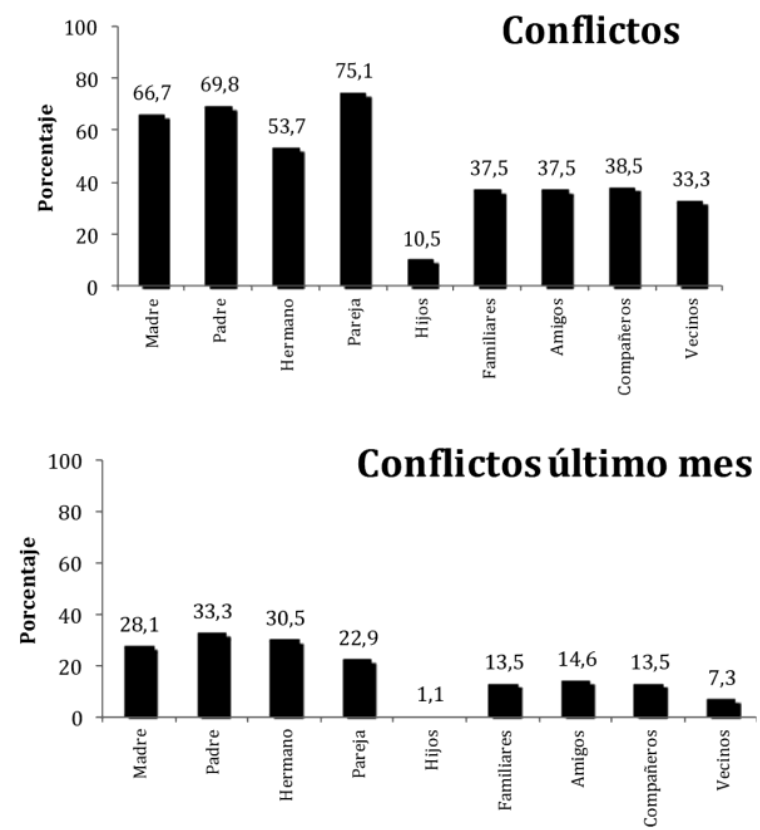

Figura 4. Porcentajes de conflictos en la muestra de usuarios recogida.
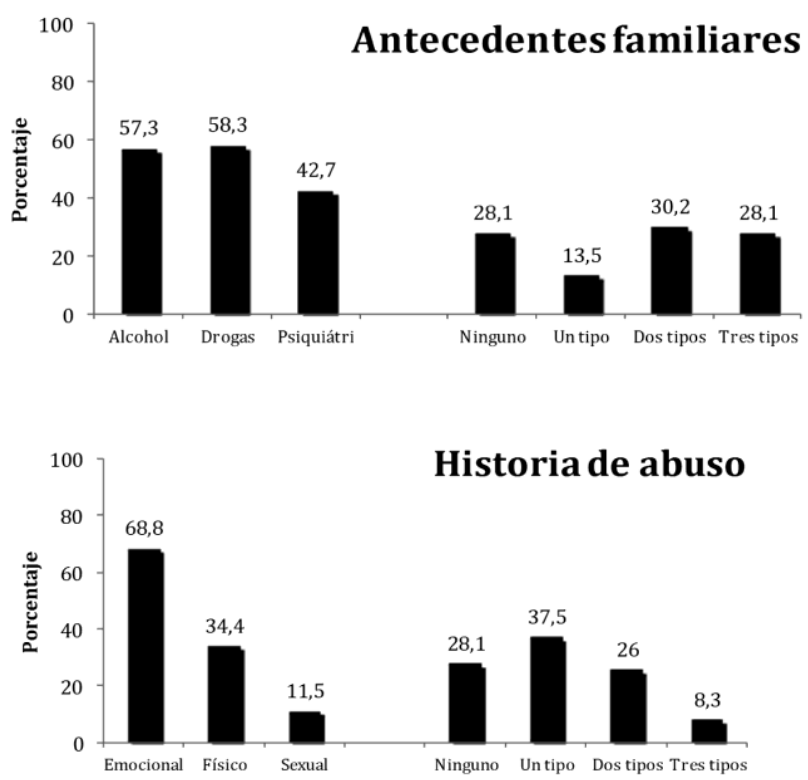

Figura 5. Porcentaje de antecedentes familiares, abusos emocionales, físicos y sexuales en la muestra de usuarios recogida.

Se han analizado algunas de las variables que aparecen significativas en los datos globales, con objeto de intentar encontrar algún tipo de relación entre ellas. En este caso, aparecen diferencias significativas en aquellos que tienen conflictos con el padre y a la vez abuso emocional o psicológico (Chi2 $=5.606, g l=1, p<.05)$, y casi significativa en el caso de abuso físico de la madre $(C h i 2=5.195, g l=1, p<.05)$. Aquellos que tienen más conflictos informan de más abuso emocional, puede que ambas variables estén señalando el mismo problema respecto a los padres. Este hecho podría venir refrendado porque también aparecen diferencias significativas en el caso de abuso emocional y mayor cantidad de antecedentes familiares (alcohol, drogas o problemas psiquiátricos) (Chi2=11.188, $g l=3, p<.01)$ y también en el caso de abuso físico y mayor cantidad de antecedentes familiares $(C h i 2=9.231, g l=3, p<.05)$. De alguna forma, con mayores conflictos con los padres, con mayores antecedentes de problemas de alcoholismo o drogas de esos padres, también mayor abuso emocional y físico. No se puede afirmar qué es causa de qué, pero son problemas muy relacionados entre sí, aunque no son determinantes para el éxito o abandono del programa. En el caso de abuso sexual no hay diferencias significativas con estas otras variables.

Dentro del consumo de diferentes drogas, en aquéllas que aparecen diferencias en cuanto abandonos, como es el caso del consumo de alcohol y de cannabis, aparece una relación significativa entre el consumo de grandes cantidades de alcohol y los antecedentes familiares de alcohol (Chi2=6.611, $g l=1, p<.01)$. En cambio, no hay relación significativa entre los antecedentes familiares (tanto del uso de alcohol, drogas o problemas psiquiátricos), y el uso de cualquier otra droga. No podría afirmarse una causa entre una situación familiar de alcoholismo y el abuso de alcohol, precisamente en los usuarios que abandonan, pero es algo que no ocurre en las demás drogas, lo que sí podría implicar algún tipo de contexto común para la adquisición de esta adicción específica en padres e hijos.

\section{Conclusiones}

El perfil psicosocial mayoritario del toxicómano adulto del Proyecto Hombre Málaga se describiría como un varón que acude al programa alrededor de los 25 años (otro grupo llega con más edad, sobre los 40 años) y que consume una media de 3 sustancias. La politoxicomanía, en gran proporción de usuarios, estaría caracterizada por el uso de cocaína, cannabis y alcohol fundamentalmente, aunque pueden consumir aún mayor cantidad de sustancias de manera simultanea en el último mes.

El grupo más joven está soltero y tienen importantes conflictos con su familia, padres y hermanos; y los mayores están casados tienen hijos y presentan los conflictos con su pareja. Pero la mayoría convive con sus padres y dice pasar la mayor parte de su tiempo con la familia.

En relación a la edad de inicio del consumo y la escalada en el tipo de droga, comienza el consumo de alcohol a los 15 años, posteriormente, el cannabis y los inhalantes. Un año más tarde el grupo de los alucinógenos, las anfetaminas, la cocaína, la heroína y, por último, un pequeño porcentaje ha tomado metadona a mayor edad. Los que comienzan más jóvenes a consumir alcohol comienzan antes otras drogas y consumen más cantidad de todas ellas. Hay una progresión 
en la edad de inicio del consumo en casi todos ellos, que comienza con pequeñas cantidades de alcohol, cannabis e inhalantes, y siguen con alucinógenos, anfetaminas y alcohol en grandes cantidades.

Atendiendo al perfil familiar, se encuentra que tienen antecedentes familiares de adicciones a alcohol y drogas, así como también antecedentes psiquiátricos en algún familiar, aunque no significativos. Cuando hay antecedentes familiares de abuso de alcohol presentan un consumo de grandes cantidades de alcohol y de cannabis.

Son familias disfuncionales en las que también ha habido abuso en la infancia. Consideran que ha sido muy frecuente el abuso emocional y, en menor medida el abuso físico. En algunos casos, especialmente en las chicas, ha habido abuso sexual. Cuanto más antecedentes familiares de consumo de tóxicos, más abuso emocional ha habido en la infancia del joven. Cuanto más conflictiva es la familia más historia de abuso físico y emocional.

El usuario del programa presenta conflictos con los familiares, padres y hermanos. Cuando llegan al Proyecto Hombre llevan un tiempo en el que los conflictos con la madre se han agudizado. Los que han comenzado más jóvenes a consumir drogas tienen más conflictos familiares. Es una de las variables más significativas, indicadas por una alta correlación, aunque no puede afirmarse si los conflictos son la fuente o la consecuencia de la conducta adictiva de los usuarios.

En relación al abandono del programa tienden a abandonar más las mujeres en la primera fase del programa y los hombres en la segunda fase. Aunque de forma global abandonan la misma proporción de hombres que de mujeres. Los que presentan más conflictos de todo tipo (familiares, pareja, vecinos, amigos, etc.) presentan un mayor abandono. Tener los conflictos con la madre es un mayor riesgo para dejar el programa.

Tienen también más probabilidad de no seguir la terapia los que consumen alcohol y cannabis, sea en pequeñas o en grandes dosis. En suma, el alcohol aumenta la probabilidad de abandonar el programa. El alta terapéutica ocurre por igual en hombres y en mujeres, independientemente del historial de consumo, edad de inicio y situación psicosocial.

Sin embargo atendiendo a las personas que con más probabilidad consiguen el éxito terapéutico o el alta, sólo se podría destacar que son en mayor proporción aquellas que apenas mantienen contacto familiar, quizás por vivir en pareja o con hijos. Al contrario de lo que podría parecer, mantener el contacto familiar, dado el alto nivel de conflictos con todos los familiares, y en muchos casos de abusos emocionales y físicos, no es precisamente un factor probable de éxito terapéutico.

\section{Discusión}

Estos resultados muestran un panorama psicosocial de las personas con adicciones que han entrado a un programa de rehabilitación de tipo residencial o comunidad terapéutica, similares a otros perfiles ya publicados en otras investigaciones. Lo importante en estos datos es la estrecha relación encontrada entre conflictos familiares, abuso emocional o físico, consumo de alcohol y abandonos del programa. Algunos estudios predictivos realizados sobre adolescentes en riesgo y con consumo de droga han señalado la historia familiar y los conflictos familiares, incluso el maltrato a los hijos, como precursores del abuso de sustancia (Beitchman et al., 1991; Dube et al., 2003; Famulary, Kinscherff y Fenton, 1992; González, 2003; Hussey y Singer, 1993; Muñoz-Rivas y Graña, 2001; Springer et al., 2007). Pero ninguno de los estudios de resultados o sobre variables predictoras ha mostrado la influencia de los conflictos familiares sobre el abandono o poco éxito de programas comunitarios como éste.

La única variable significativa para las altas terapéuticas es precisamente tener poco contacto con la familia. Esto debería hacer reflexionar sobre los factores en los que están incidiendo las actividades del programa de rehabilitación, pues habría de tener en cuenta estos conflictos para resolverlos. Ya se trate de conflictos anteriores al problema de adicción o bien como consecuencia de ella, son los factores que pueden estar manteniendo un gran malestar del usuario que haga que abandone finalmente el programa. Podría aconsejarse, pues, que se integrase a los familiares más directos en el programa, pero no como "acompañantes" de la abstinencia sino como "parte del problema", quizás enseñándoles cómo comportarse, cómo reaccionar, cómo interaccionar de una forma más positiva, intentando disminuir o eliminar esa fuente de conflicto. También integrando otros factores como habilidades sociales, resolución de problemas, mediación familiar, autonomía personal, búsqueda de independencia, e incluso habilidades para la búsqueda de empleo y formación profesional que le permita una vida independiente.

Secades y Fernández (2000) a partir de sus estudios sobre la retención en los programas de comunidad terapéutica, realizaron ya una serie de recomendaciones para mejorar esas tasas de retención en el programa, referidas tanto a los usuarios (restringir criterios de inclusión, incremento en la motivación inicial para adherirse al programa), como los referidos al propio programa (adaptarse a las necesidad individuales de cada grupo de personas con adicciones diferentes, implicar a personas allegadas, terapias de incentivo y manejo de contingencias, y también combinar terapias farmacológicas y psicológicas), y respecto a los terapeutas (mejorar la formación, incentivar, motivar, y tener una continuidad y estabilidad en el tratamiento). Quizás sería necesario realizar estudios más especializados sobre los factores que contribuyen a esos abandonos; pero sería aún más interesante ahondar en los factores que propician las altas, que aunque van aumentando progresivamente con los años, podrían incrementarse aún más si se conociese cuáles son esos factores protectores en la misma familia, los factores motivacionales y de tratamiento de los terapeutas, los factores de inserción social mediante empleo e independencia económica, tal como ha señalado la revisión de estudios sobre factores predictivos (Girón, 2007). 
Para obtener conclusiones definitivas también hay que considerar las limitaciones de este estudio como se señalaban al principio, por un lado, el hecho de que todos los datos proceden de entrevistas o cuestionarios, es decir, información suministrada por los propios usuarios, pero no contrastada con otras fuentes de información o con otras técnicas más objetivas, como serían observaciones directas o pruebas biomédicas; y por otro, que los resultados se basan en una parte de toda la muestra posible de usuarios que pasan por el programa, dado el tipo de estudio post-facto y las dificultades para recoger historiales de todos los usuarios. Una muestra más amplia habría permitido un mejor análisis estadístico de tipo predictivo.

\section{Referencias}

Arbour, S., Hambley, J., y Ho, V. (2011). Predictors and outcome of aftercare participation of alcohol and rug users completing residential treatment. Substance Use and Misuse, 46 (10), 1275-1287.

Beitchman, H.J., Zucher, J.K., Hood, E.J., Da Costa, A.G. y Ackman, D. (1991).A review of the long-term effects of child sexual abuse. Child Abuse and Neglect, 16, 537-556.

Bobes, J., Bascarán, M.T., Bobes, M.T., Carballo, J.L., Díaz, E.M., Flores, E., García-Portilla, M.P., Sáinz, P.A. (2007).Valoración de la gravedad de la adicción: Aplicación a la gestión clínica y monitorización de los tratamientos. Madrid: Ministerio de Sanidad y Consumo. (Consultada el 10-12-2009). Monográfico disponible en http://www.pnsd.msc.es/Categoria2/publica/pdf/ValoracionGra vedadAdiccion.pdf

Cardenal, V., Moreno, P., y Ortiz-Tallo, M. (1994). Evaluación cualitativa del centro de rehabilitación de toxicómanos de Proyecto Hombre. En V. Cardenal., J. Corbalán, R. Esteve, V. Gradillas, B. Moreno, P. Moreno, M. Navarro., Ortiz-Tallo, M., y L. Valero (eds). Evaluación de programas de reinserción social. Málaga: Miguel Gómez Ediciones (pags. 125-159).

Crevecoeur, M.D., Ransom, L., Myers, A.C., Annon, J.J., Diep, N., Gonzales, R., Rawson, R.A., Viemes, J., Sugita, W., y Barger, J. (2010) Inside the black box: Measuring addiction treatment services and their relation to outcomes. Journal of Psychoactive Drugs, 6, 269-276.

Dube, S. R., Anda, R. F., Felitti, V. J., Chapman, D., Williamson, D. F., \& Giles, W. H. (2001). Childhood abuse, household dysfunction and the risk of attempted suicide throughout the life span: Findings from the Adverse Childhood Experiences Study. Journal of the American Medical Association, 286, 3089-3096.

Evans, E., Li, L.B., y Hser, Y.I. (2008). Client and program factors associated with dropout from court mandated drug treatment. Evaluation and Program Planning, 32 (3), 204-212.

Famulary, R., Kinscherff, R., y Fenton, T. (1992) Parental substance abuse and the nature of child maltreatment. Cbild Abuse and Neglect, 16 (4), 475-483.

Fernández-Hermida, J.R., Secades, R. (2001). Evaluación de la eficacia del programa. Madrid: Proyecto Hombre.

Fernández-Hermida, J.R., Secades, R., Magdalena-Benavente, Y., Riestra del Rosal, C. (1999). Evaluación de la eficacia del programa educativoterapéutico para rehabilitación de toxicómanos de Proyecto Hombre en Asturias. Oviedo: Servicio Central de Publicaciones del Principado de Asturias.

Fernandez-Montalvo, J., López-Goñi, J.J., Illescas, C., Landa, N., y Lorea, I. (2008). Evaluation of a therapeutic community treatment program: A long-term follow-up study in Spain. Sustance Use \& Misuse, 43 (10), 1362-1377.
Con todo, creemos que la muestra es representativa de los usuarios del programa y las conclusiones generales, sobre los factores personales y familiares que inciden en los abandonos del programa (historia y conflictos familiares, policonsumo, alcohol, abuso emocional o físico), pueden ayudar a mejorar en un futuro las estrategias de tratamiento e integración que se realizan dentro de este tipo de programas de rehabilitación en comunidad terapéutica. $\mathrm{Al}$ menos, estos resultados han de considerarse un factor de riesgo al que habría que prestar especial atención cuando los usuarios acceden por primera vez al programa, para trabajar sobre ellos y que la tasa de abandonos fuese menor cada año.

Girón, S. (2007). Los estudios de seguimiento en drogodependencias: Una aproximación al estado de la cuestión. Trastornos Adictivos, 9 (2), 75-96

González, J.M. (2003) Abuso sexual infantil. Un estudio de sus consecuencias en mujeres alcohólicas y fármaco dependientes en $\mathrm{Ba}$ rranquilla, Colombia. Revista Electrónica de Psicología Científica. (Consultada el 10-12-2009). Disponible en http://www.psicologiacientifica.com

Hambley, J., Arbour, S., y Sivagnanasundaram, L. (2010). Comparing outcomes for alcohol and drug abuse clients: A 6-month follow-up of clients who completed a residential treatment programme. Jour nal of Substance Use, 15 (3), 184-200.

Hussey, D. y Singer, M. (1993). Psychological distress, problem behaviors and family functioning of sexually abused adolescent inpatients. Journal of the American Academy of Child and Adolescent Psychiatry, 32 (5), 954-961

López-Goñi, J.J., Fernández-Montalvo, J., Illescas, C., Landa, N., y Lorea, I. (2008a). Razones para el abandono del tratamiento en una comunidad terapéutica. Trastornos Adictivos, 10 (2), 104-111.

López-Goñi, J.J., Fernández-Montalvo, J., Illescas, C., Landa, N., y Lorea, I. (2008b). Determining socio-demographic predictors of treatment dropouts: results in a therapeutic community. International Journal of Social Welfare, 17 (4), 374-378.

Mulder, R.T., Frampton, C.M.A., Peka, H., Hampton, G., Masters, T. (2009). Predictor of 3-month retention in a drug treatment therapeutic community. Drug and Alcohol Review, 28 (4), 366-371.

Muñoz-Rivas, M. y Graña López, J.L. (2001). Factores familiares de riesgo y protección para el consumo de drogas en adolescentes. Psicothema, 13 (1), 87-94.

Rodríguez de Fonseca, F., Alexandre, R., Cami, J., Navarro, M., Torrerns, M., Iraurgf, J., Oñorbe, J., De Andrés, R., Colom, J., Tomás, S., y Molina, M. (2006). La investigación en drogodependencias. Trastornos Adictivos, 8 (2), 115-132.

Sánchez-Hervás, E., Secades-Villa, R., Santoja-Gómez, F.J., ZacarésRomaguera, R., García-Rodríguez, O., Martín-Yañez, E., Catalatyud-Francés, M., García-Fernández, G. (2010). Abandono del tratamiento en adictos a la cocaína. Adicciones, 22 (1), 59-64.

Secades, R., y Benavente, Y. (2000). Predictores de la retención en una comunidad terapéutica para drogodependientes. Adicciones, 12 (3), 365-371.

Secades, R., y Fernández, J.R. (2000). Cómo mejorar las tasas de retención en los tratamientos de drogodependencias. Adicciones, 12 (3), 353-363.

Secades, R., y García, O. (2005). Estudio de la percepción de la gravedad del uso de drogas en familias con hijos adolescentes. Madrid: Asociación Proyecto Hombre. (Consultada el 10-12-2009). Mono- 
grafía disponible en http://www.observatoripalma.org/imgdb//archivo_doc7824.pdf Sociedad Española de Toxicomanías (2006). Documento de consenso de la SET sobre la importancia de la investigación en drogodependencias. Trastornos Adictivos, 8 (2), 133-134.

Springer, K. W., Sheridan, J., Kuo, D., \& Carnes, M. (2007). Long-term physical and mental health consequences of childhood physical abuse: Results from a large population-based sample of men and women. Child Abuse and Neglect, 31, 517-53.
Vanderplasschen, W., Bloor, M., McKeganey, N. (2010). Long-term outcomes of aftercare participation following various forms of drug abuse treatment in Scotlan. Journal of Drug Issues, 40 (3), 703728.

White, E., Browne, C., McKiernan, B., Sweeney, B. (2010). Keltoi rehabilitation programme: Post-discharge outcome study. Drugs Education Prevention and Policy, 18 (5), 353-360.

(Artículo recibido: 14-01-2011, revisión: 23-10-2011, aceptado: 8-01-2012) 